\title{
HEXACARBOCYCLIC TRITERPENES FROM LEAVES OF CRYPTOMERIA JAPONICA
}

\author{
Wen-Chiung Su, Jim-Min Fang and Yu-Shia Cheng* \\ Department of Chemistry, National Taiwan University, Taipei, Taiwan 10617, Republic of China
}

(Received in revised form 19 January 1993)

Key Word Index-Cryptomeria japonica; Taxodiaceae; leaves, hexacarbocyclic triterpenes; chamaecydin; 6-hydroxychamaecydins; $10^{\prime}$-hydroxycryptoquinones.

\begin{abstract}
Five hexacarbocyclic triterpenes, namely chamaecydin, $6 \alpha$-hydroxychamaecydin, $6 \beta$-hydroxychamaecydin, $10^{\prime} \alpha$-hydroxycryptoquinone and $10^{\prime} \beta$-hydroxycryptoquinone, whose skeleton comprises spiro-annulation of abietanetype diterpenes and thujane-type monoterpenes, were isolated from the leaves of Cryptomeria japonica.
\end{abstract}

\section{INTRODUCTION}

The Japanese cedar, Cryptomeria japonica D. Don., is a widely distributed conifer called 'sugi' in Japanese [1]. Its constituents have been extensively studied. The leaves are reported to contain monoterpenes, diterpenes and flavones [2-9]. In this paper, we report five uncommon triterpenes 1-5, isolated from the ethyl acetate-soluble part of the leaves of $C$. japonica.

\section{RESULTS AND DISCUSSION}

The molecular formula $\mathrm{C}_{30} \mathrm{H}_{40} \mathrm{O}_{3}$ of 1 was deduced from its exact mass [M] ${ }^{+}$at $m / z$ 448.298. The ${ }^{13} \mathrm{C}$ NMR spectrum (Table 1) showed two carbonyl groups $(\delta 205.7$ and 182.6) and three double bonds $(\delta 123.6,136.3,144.6$, 145.1, 149.1 and 151.7). These analyses indicated that 1 , mp $197-198^{\circ},[\alpha]_{\mathrm{D}}^{25}+33^{\circ}\left(\mathrm{CHCl}_{3} ; c\right.$ 2.5), is an uncommon hexacarbocylic triterpene, chamaecydin, previously isolated from the seed of Chamaecyparis obtusa (Cupressaceae) [10]. Compound 1 contains two subunits, an abietane-type diterpene and a thujane-type monoterpene, which are spiro-annulated. Assignment of the proton and carbon resonances (Tables 1 and 2) was further supported by a $\mathrm{C}-\mathrm{H}$ COLOC spectrum and the NOE experiments (Fig. A). For example, the quaternary C-4' at $\delta 57.9$ was shown to correlate with $\mathbf{H}-2^{\prime}$ and $\mathbf{H}-6^{\prime}$. Irradiation of the $\mathrm{H}-15$ resonance at $\delta 3.16$ caused NOEs on the two cyclopropane methylene protons. In the CD spectrum, compound 1 exhibited a distinct positive Cotton effect with the maximum at $455 \mathrm{~nm}$ in addition to others.

Compound $2\left(\mathrm{C}_{30} \mathrm{H}_{40} \mathrm{O}_{4}\right), \mathrm{mp} 220-222^{\circ},[\alpha]_{D}^{25}-51^{\circ}$ $\left(\mathrm{CHCl}_{3} ; c 0.8\right)$, was similarly determined to be $6 \alpha$-hydrox-

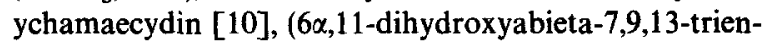

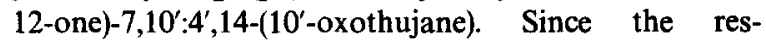

*Author to whom correspondence should be addressed. onance of H-6 at $\delta 5.02$ showed a large coupling constant $\left(J_{5.6}=10 \mathrm{~Hz}\right.$ ), the H-6 should be on the axial position (the $\beta$-face). The IR absorptions at 1693 and $1608 \mathrm{~cm}^{-1}$ as well as the UV absorption at $350 \mathrm{~nm}(\varepsilon 25000)$ were characteristics of the quinone methide moiety of 2 .

By analyses of the spectral properties, compound 3 $\left(\mathrm{C}_{30} \mathrm{H}_{40} \mathrm{O}_{4}\right)$ was readily recognized as $6 \beta$-hydroxychamaecydin, an epimer of 2 . The equatorial $6 \alpha$-proton occurred at $\delta 5.10$ with a small coupling constant, $J_{5,6}=3.5 \mathrm{~Hz}$. The new triterpene 3 exhibited a positive Cotton effect with the maximum at $453 \mathrm{~nm}$, whereas 2 showed a negative Cotton effect with the minimum at $457 \mathrm{~nm}$.

Compound 4 showed the exact mass $[\mathrm{M}]^{+}$at $\mathrm{m} / \mathrm{z}$ 464.292 , indicating its molecular formula $\mathrm{C}_{30} \mathrm{H}_{40} \mathrm{O}_{4}$. The presence of intramolecular hydrogen-bonded hydroxyl group was inferred from the IR absorption at $3298 \mathrm{~cm}^{-1}$ (sharp). The hydroxyl resonance at $\delta 7.66$ in the NMR spectrum was not shifted by changing to a different concentration of sample. The IR spectrum also displayed strong absorptions at 1650 and $1637 \mathrm{~cm}^{-1}$ attributable to a conjugated carbonyl group and a quinone moiety, respectively. Analysis of the ${ }^{1} \mathrm{H}$ NMR spectrum (Table 2) revealed two isopropyl groups and three methyl groups positioned on tertiary carbons. Two methylene protons of a cyclopropane ring occurred at $\delta 0.57$ and 0.83 , but no olefinic protons were present. The ${ }^{13} \mathrm{C}$ NMR spectrum exhibited a resonance at $\delta 191.8$ for a ketone, a resonance at $\delta 183.1$ for a quinone and the signals for six olefinic carbons bearing no proton. Judging from the above spectral properties, we suggest that 4 is a hexacyclic triterpene (11-hydroxyabieta-7,9,13-triene-6,12-dione)$7,10^{\prime}: 4^{\prime}, 14-\left(10^{\prime} \beta\right.$-hydroxythujane), constructed by two subunits of the abietane-type diterpene and the thujanetype monoterpene. These two subunits were spiro-annulated $\left(7,10^{\prime}: 14,4^{\prime}\right)$ to form an extra carbocyclic ring. The $\lambda_{\max }$ at $354 \mathrm{~nm}$ ( 829800 ) was in agreement with the calculated value of a vinylogous quinone moiety. Irradiation of $\mathrm{H}-10^{\prime}(\delta 4.70)$ geminal to the hydroxyl group 


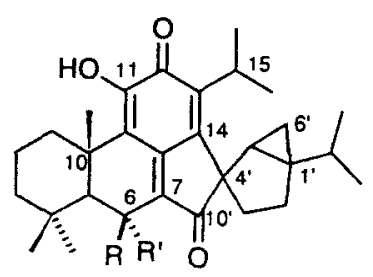

$1 \mathrm{R}=\mathrm{R}^{\prime}=\mathrm{H}$

$2 \mathrm{R}=\mathrm{H}, \mathrm{R}^{\prime}=\mathrm{OH}$

$3 R=O H, R^{\prime}=H$

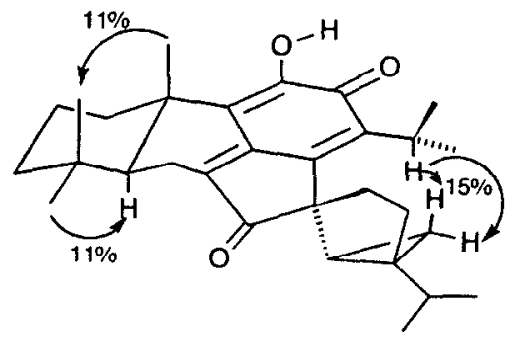

A

Table 1. ${ }^{13} \mathrm{CNMR}$ spectral data of compounds 1-4 and 6 $\left(\mathrm{CDCl}_{3} \text { solution, } \delta \text { values in } \mathrm{ppm}\right)^{*}$

\begin{tabular}{|c|c|c|c|c|c|}
\hline C & 1 & 2 & 3 & 4 & 6 \\
\hline 1 & 36.5 & 37.2 & 37.9 & 36.9 & 36.9 \\
\hline 2 & 18.6 & 18.6 & 18.9 & 18.4 & 18.4 \\
\hline 3 & 41.6 & 43.2 & 43.9 & 42.7 & 42.7 \\
\hline 4 & 33.8 & 34.2 & 34.7 & 33.6 & 35.3 \\
\hline 5 & 50.8 & 56.1 & 53.3 & 63.7 & 64.4 \\
\hline 6 & 19.4 & 67.5 & 60.6 & 191.8 & 195.6 \\
\hline 7 & 145.1 & 144.5 & 145.7 & 144.5 & 146.3 \\
\hline 8 & 151.7 & 150.9 & 151.7 & 156.8 & 158.1 \\
\hline 9 & 123.6 & 122.8 & 123.9 & 121.8 & 122.3 \\
\hline 10 & 38.6 & 40.2 & 38.7 & 43.6 & 43.3 \\
\hline 11 & 144.6 & 142.9 & 142.1 & 143.5 & 140.4 \\
\hline 12 & 182.6 & 182.8 & 182.4 & 183.1 & 181.8 \\
\hline 13 & 136.3 & 137.7 & 137.9 & 140.0 & 129.2 \\
\hline 14 & 149.4 & 148.9 & 149.8 & 144.8 & 150.9 \\
\hline 15 & 28.7 & 28.9 & 28.9 & 28.5 & 29.1 \\
\hline 16 & 20.0 & 20.1 & 20.1 & 20.3 & 20.4 \\
\hline 17 & 20.4 & 20.4 & 20.5 & 20.5 & 20.6 \\
\hline 18 & 33.4 & 36.4 & 33.4 & 33.5 & 33.3 \\
\hline 19 & 22.0 & 22.7 & 24.1 & 22.5 & 22.2 \\
\hline 20 & 19.1 & 21.3 & 21.9 & 21.9 & 21.8 \\
\hline $1^{\prime}$ & 34.7 & 34.8 & 34.8 & 32.9 & 33.1 \\
\hline $2^{\prime}$ & 30.7 & 30.7 & 30.3 & 32.1 & 31.1 \\
\hline $3^{\prime}$ & 29.8 & 29.7 & 29.7 & 26.3 & 30.0 \\
\hline $4^{\prime}$ & 57.9 & 58.4 & 58.0 & 59.8 & 58.7 \\
\hline $5^{\prime}$ & 30.9 & 31.0 & 31.4 & 35.0 & 31.3 \\
\hline $6^{\prime}$ & 13.2 & 13.4 & 13.6 & 14.2 & 13.0 \\
\hline $7^{\prime}$ & 31.6 & 31.6 & 31.8 & 28.6 & 30.7 \\
\hline $8^{\prime}$ & 19.6 & 19.7 & 19.8 & 20.0 & 19.6 \\
\hline $9^{\prime}$ & 20.2 & 20.2 & 20.2 & 20.1 & 20.2 \\
\hline $10^{\prime}$ & 205.7 & 207.4 & 206.1 & 80.2 & 200.7 \\
\hline
\end{tabular}

${ }^{*}$ Owing to small amount of 5 available, its ${ }^{13} \mathrm{C}$ signals were not assigned.

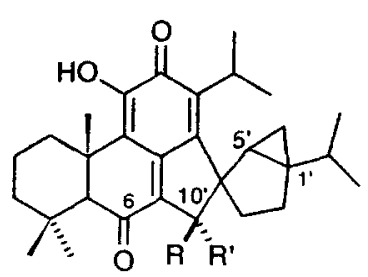
$4 \mathrm{R}=\mathrm{OH}, \mathrm{R}^{\prime}=\mathrm{H}$
$5 \mathrm{R}=\mathrm{H}, \mathrm{R}^{\prime}=\mathrm{OH}$
$6 \mathrm{~A}, \mathrm{R}^{\prime}=0$

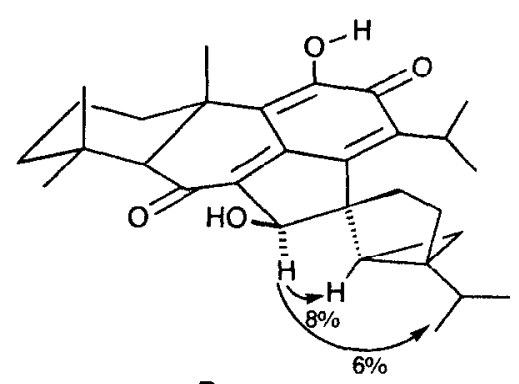

B

caused NOEs of $\mathrm{H}-5^{\prime}$ and the resonances of $\mathrm{C}-1^{\prime}$ isopropyl groups (Fig. B). Thus, $\mathrm{H}-10^{\prime}$ is $\alpha$-orientated. The absolute configuration is assigned $\left(5 S, 10 S, 1^{\prime} R, 5^{\prime} S\right)$ by analogy to that of 1 . The novel compound 4 is named as $10^{\prime} \beta$-hydroxycryptoquinone, of which 'crypto' implies the plant source of Cryptomeria genus and the structural feature of the hidden (crypt) quinone.

The C-10' epimer 5, $10^{\prime} \alpha$-hydroxycryptoquinone, was isolated in minute amount. Its structure was deduced from spectral analyses and confirmed by a chemical correlation. Thus, oxidation of 4 and 5 with $\mathrm{MnO}_{2}$, respectively, gave the same ketone $6,10^{\prime}$-oxocryptoquinone. Compound 5 is more polar than 4 .

In summary, we isolated chamaecydin (1), $6 \alpha$-hydroxychamaecydin (2) and three new analogues, $6 \beta$-hydroxychamaecydin (3), 10' $\beta$-hydroxycryploquinone (4) and $10^{\prime} \alpha$-hydroxycryptoquinone (5) from the leaves of Cryptomeria japonica. These uncommon hexacarbocyclic triterpenes are constructed by the spiro-annulation of abietane-type diterpenes and thujane-type monoterpenes. The biological significance and possible pharmacological effects related to their extended vinylogous quinone moieties await further investigation.

\section{EXPERIMENTAL}

General. Merck silica gel $60 \mathrm{~F}$ sheets were used for analyt. TLC. HPLC was carried out on a Hibar Lichrosorb Si $60(7 \mu \mathrm{m})$ column $(25 \mathrm{~cm} \times 1 \mathrm{~cm})$.

Plant material. The plant used in this study was introduced from Japan and cultivated in suburban Taipei. A voucher specimen is deposited in our laboratory. The leaves $(1.4 \mathrm{~kg})$ of $C$. japonica $\mathrm{D}$. Don. were exhaustively extracted with $\mathrm{Me}_{2} \mathrm{CO}$. The extract was passed through a 
Table 2. ${ }^{1} \mathrm{HNMR}$ spectral data of compounds $1-6\left(\mathrm{CDCl}_{3}\right.$ solution, $\delta$ values in ppm, $J$ values in $\left.\mathrm{Hz}\right)$

\begin{tabular}{|c|c|c|c|c|c|c|}
\hline $\mathbf{H}$ & $\mathbf{1}$ & 2 & 3 & 4 & $\mathbf{s}$ & 6 \\
\hline $1 \alpha$ & $\begin{array}{l}1.56(d d d, J \\
13,12,3.5)\end{array}$ & $\begin{array}{l}1.55(d d d, J \\
12,12,3)\end{array}$ & $\begin{array}{l}1.45(d d d, J \\
13,13,4)\end{array}$ & $\begin{array}{l}1.73(d d d, J \\
12,11,3)\end{array}$ & $1.69(\mathrm{~m})$ & $1.70(\mathrm{~m})$ \\
\hline $1 \beta$ & $\begin{array}{l}2.82(d d d, J \\
13,4,1.5)\end{array}$ & $\begin{array}{l}2.73(d d d, J \\
12,4,1.5)\end{array}$ & $\begin{array}{l}2.83(d d d, J \\
13,4,1)\end{array}$ & $\begin{array}{l}2.79(d d d, J \\
11,4,1)\end{array}$ & $\begin{array}{l}2.72(d d d, J \\
12,4,1)\end{array}$ & $\begin{array}{l}2.80(d d d, J \\
11,4,1)\end{array}$ \\
\hline $2 x$ & $1.60(\mathrm{~m})$ & $1.56(m)$ & $1.57(\mathrm{~m})$ & $1.60(m)$ & $1.58(m)$ & $1.60(m)$ \\
\hline $2 \beta$ & $1.64(m)$ & $1.61(m)$ & $1.82(m)$ & $1.80(m)$ & $1.79(\mathrm{~m})$ & $1.80(m)$ \\
\hline $3 \alpha$ & $\begin{array}{l}1.21(d d d, J \\
12,12,2)\end{array}$ & $\begin{array}{l}1.40(d d d, J \\
12,12,2)\end{array}$ & $\begin{array}{l}1.23(d d d, J \\
12,12,3)\end{array}$ & $\begin{array}{l}1.21(d d d, J \\
12,12,3)\end{array}$ & $\begin{array}{l}1.21(d d d, J \\
12,12,3)\end{array}$ & $\begin{array}{l}1.71(d d d, J \\
12,12,2)\end{array}$ \\
\hline $3 \beta$ & $\begin{array}{l}1.46(d d d, J \\
12,4.5,2.5)\end{array}$ & $\begin{array}{l}1.54(d d d, J \\
12,4.5,2.5)\end{array}$ & $\begin{array}{l}1.40(d d d, J \\
12,4,2)\end{array}$ & $\begin{array}{l}1.41(d d d, J \\
12,4,1)\end{array}$ & $\begin{array}{l}1.40(d d d, J \\
12,4,1)\end{array}$ & $\begin{array}{l}1.40(d d d, J \\
12,4,2)\end{array}$ \\
\hline 5 & $\begin{array}{l}1.49(d d, J 12 \\
3.5)\end{array}$ & $1.69(d, J 10)$ & $1.35(d, J 3.5)$ & $2.64(s)$ & $2.62(s)$ & $2.58(s)$ \\
\hline $6 x$ & $\begin{array}{l}2.69(d d, J 19, \\
3.5)\end{array}$ & & $5.10(d, J 3.5)$ & & & \\
\hline $6 \beta$ & $\begin{array}{l}2.18(d d, J 19 \\
12)\end{array}$ & $5.02(d, J 10)$ & & & & \\
\hline 15 & $\begin{array}{l}3.16(\text { sept }, J \\
7)\end{array}$ & $\begin{array}{l}3.16(s e p t, J \\
7)\end{array}$ & $\begin{array}{l}3.17 \text { (sept, } J \\
7)\end{array}$ & $3.32($ sept,$J 7)$ & $\begin{array}{l}3.56(\text { sept }, J \\
7)\end{array}$ & $\begin{array}{l}3.26 \text { (sept, J } \\
7)\end{array}$ \\
\hline 16 & $1.26(d, J 7)$ & $1.26(d, J 7)$ & $1.27(d, J 7)$ & $1.27(d, J 7)$ & $1.24(d, J 7)$ & $1.25(d, J 7)$ \\
\hline 17 & $1.28(d, J 7)$ & $1.28(d, J 7)$ & $1.29(d, J 7)$ & $1.28(d, J 7)$ & $1.27(d, J 7)$ & $1.30(d, J 7)$ \\
\hline 18 & $0.94(s)$ & $1.13(s)$ & $1.10(s)$ & $1.14(s)$ & $1.14(s)$ & $1.11(s)$ \\
\hline 19 & $0.97(s)$ & $1.16(s)$ & $1.33(s)$ & $1.26(s)$ & $1.24(s)$ & $1.28(s)$ \\
\hline 20 & $1.14(s)$ & $1.20(s)$ & $1.52(s)$ & $1.26(s)$ & $1.25(\mathrm{~s})$ & $1.30(s)$ \\
\hline $2^{\prime} \alpha$ & $\begin{array}{l}1.70(d d d d, J \\
12,8,2,1)\end{array}$ & $\begin{array}{l}1.67(d d d d, J \\
12,8,2,1)\end{array}$ & $\begin{array}{l}1.80(\text { dddd, } J \\
12,8,1,1)\end{array}$ & $\begin{array}{l}1.91(\text { dddd, } J \\
12,7.5,2.5 \\
\text { 1) }\end{array}$ & $\begin{array}{l}1.82 \text { (dddd, } J \\
12,7.5,2.5 \\
1)\end{array}$ & $\begin{array}{l}1.73(d d d, J \\
12,8,1.5)\end{array}$ \\
\hline $2^{\prime} \beta$ & $\begin{array}{l}1.67\left(d d d d_{,} J\right. \\
12,7,1.5)\end{array}$ & $\begin{array}{l}1.66(d d d, J \\
12,7,1.5)\end{array}$ & $\begin{array}{l}1.70(d d d, J \\
12,7,1.5)\end{array}$ & $\begin{array}{l}1.52(d d d, J \\
12,7.5,1)\end{array}$ & $\begin{array}{l}1.43(d d d, J \\
12,7.5,1)\end{array}$ & $\begin{array}{l}1.68(d d d, J \\
12,7,2)\end{array}$ \\
\hline $3^{\prime} \alpha$ & $\begin{array}{l}2.24(d d d, J \\
12,8,1.5)\end{array}$ & $\begin{array}{l}2.20(d d d, J \\
12,8,1.5)\end{array}$ & $\begin{array}{l}2.27(d d d, J \\
12,8,1.5)\end{array}$ & $\begin{array}{l}2.18(d d d, J \\
12,7.5,1)\end{array}$ & $\begin{array}{l}2.02(d d d, J \\
12,7.5,1)\end{array}$ & $\begin{array}{l}2.25(d d d, J \\
12,8,2)\end{array}$ \\
\hline $3^{\prime} \beta$ & $\begin{array}{l}1.81(d d d, J \\
12,7,2)\end{array}$ & $\begin{array}{l}1.83(d d d, J \\
12,7,2)\end{array}$ & $\begin{array}{l}1.83(d d d, J \\
12,7,1)\end{array}$ & $\begin{array}{l}1.66(d d d, J \\
12,7.5,2.5)\end{array}$ & $\begin{array}{l}1.64(d d d, J \\
12,7.5,2.5)\end{array}$ & $\begin{array}{l}1.83(d d d, J \\
12,7,1.5)\end{array}$ \\
\hline $5^{\prime}$ & $\begin{array}{l}1.02(d d, J 8 \\
4)\end{array}$ & $\begin{array}{l}1.08(d d, J 8 \text {, } \\
\text { 4) }\end{array}$ & $\begin{array}{l}1.05(d d, J 8 \\
\text { 4) }\end{array}$ & $\begin{array}{l}0.96(d d, J 8 \\
4)\end{array}$ & $\begin{array}{l}0.84(d d, J 8 \\
4)\end{array}$ & $\begin{array}{l}1.10(d d, J 8 \text {, } \\
\text { 4) }\end{array}$ \\
\hline $6^{\prime} \alpha$ & $\begin{array}{l}0.69(d d d, J 8 \\
6,1)\end{array}$ & $\begin{array}{l}0.65(d d d, J \\
8,6,1)\end{array}$ & $\begin{array}{l}0.70(d d d, J 8 \\
6,1)\end{array}$ & $\begin{array}{l}0.57(d d d, J 8 \\
6,1)\end{array}$ & $\begin{array}{l}0.74(d d d, J 8 \\
6,1)\end{array}$ & $\begin{array}{l}0.84(d d, J 8, \\
6)\end{array}$ \\
\hline $6^{\prime} \beta$ & $\begin{array}{l}0.75(d d, J 6 \\
4)\end{array}$ & $\begin{array}{l}0.81(d d, J 6 \\
\text { 4) }\end{array}$ & $\begin{array}{l}0.78(d d, J 6 \\
4)\end{array}$ & $\begin{array}{l}0.83(d d, J 6 \\
4)\end{array}$ & $\begin{array}{l}0.76(d d, J 6 \\
4)\end{array}$ & $\begin{array}{l}0.89(d d, J 6 \text {, } \\
\text { 4) }\end{array}$ \\
\hline 7 & $\begin{array}{l}1.78(\text { sept }, J \\
7)\end{array}$ & $\begin{array}{l}1.77 \text { (sept, } J \\
7)\end{array}$ & $\begin{array}{l}1.71 \text { (sept, } J \\
7)\end{array}$ & $1.70($ sept,$J 7)$ & $\begin{array}{l}1.70(\text { sept, J } \\
7)\end{array}$ & $\begin{array}{l}1.81(\text { sept }, J \\
7)\end{array}$ \\
\hline $8^{x}$ & $0.78(d, J 7)$ & $0.78(d, J 7)$ & $0.80(d, J 7)$ & $0.86(d, J 7)$ & $0.85(d, J 7)$ & $0.75(d, J 7)$ \\
\hline $\begin{array}{l}9 \\
10^{\prime}\end{array}$ & $1.03(d, J 7)$ & $1.01(d, J 7)$ & $1.03(d, J 7)$ & $\begin{array}{l}0.99(d, J 7) \\
4.70(s)\end{array}$ & $\begin{array}{l}1.00(d, J 7) \\
4.60(s)\end{array}$ & $1.02(d, J 7)$ \\
\hline $11-\mathrm{OH}$ & $7.71(s)$ & $7.75(s)$ & $7.75(\mathrm{~s})$ & $7.66(s)$ & $7.63(s)$ & $7.93(n)$ \\
\hline
\end{tabular}

pad of charcoal, concd and re-extracted with EtOAc. The EtOAc-soluble portion ( $45 \mathrm{~g}$ ) was chromatographed on a silica gel column by elution with gradients of hexane and EtOAc. The appropriate frs were combined and purified by HPLC to give 1 ( $30 \mathrm{mg}), 2(8 \mathrm{mg}), 3(8 \mathrm{mg}), 4(8 \mathrm{mg})$ and 5 (4 mg).

Chamaecydin (1). Orange crystals. Mp 197-198 (from hexane). $[\alpha]_{\mathrm{D}}^{25}+33^{\circ}\left(\mathrm{CHCl}_{3} ; c 2.5\right)$. TLC $(0.7 \%$ EtOAc in hexane) $R_{f} 0.16$. IR $v_{\max }^{\mathrm{KBr}} \mathrm{cm}^{-1}: 3273,1693,1611,1389$, 1370. UV $\lambda_{\max }^{\mathrm{CHCl}_{3}} \mathrm{~nm}(\varepsilon): 352$ (32600), 342 (25400), 336 (27 800), $282(2130), 240(12300) . \mathrm{CD}\left(\mathrm{CHCl}_{3}\right):[\theta]_{474}$ $+1010,[\theta]_{455}+1480,[\theta]_{430}-1050,[\theta]_{410}+630,[\theta]_{393}$ $-620,[\theta]_{375}+710,[\theta]_{357}-600,[\theta]_{337}+960,[\theta]_{316}$ $-460,[\theta]_{290}+420,[\theta]_{267}-190,[\theta]_{253}+80,[\theta]_{231}$ -1170 . EIMS (70 eV) $m / z$ (rel. int.) $448[\mathrm{M}]^{+}(100), 433$ (10), 405 (64), 377 (9), $366(16), 352$ (13), 340 (18), 281 (14). HRMS for $\mathrm{C}_{30} \mathrm{H}_{40} \mathrm{O}_{3}$ requires 448.2979. Found 448.2976.

6 $\alpha$-Hydroxychamaecydin (2). Orange crystals. Mp 220-222 (from hexane). $[\alpha]_{\mathrm{D}}^{25}-51^{\circ}\left(\mathrm{CHCl}_{3} ; c 0.8\right)$. TLC (0.7\% EtOAc in hexane) $R_{f} 0.13$. IR $v_{\max }^{\mathrm{XBr}} \mathrm{cm}^{-1}: 3529$, $3303,1693,1608,1380,1372$. UV $\lambda_{\max }^{\mathrm{CHCl}_{3}} \mathrm{~nm}(\varepsilon): 350$ (25000), $341(21600), 335(22000), 274(2300), 241(9300)$. $\mathrm{CD}\left(\mathrm{CHCl}_{3}\right):[\theta]_{457}-990,[\theta]_{434}+80,[\theta]_{411}-720$, $[\theta]_{381}+60,[\theta]_{362}-110,[\theta]_{345}+50,[\theta]_{323}-480$, $[\theta]_{301}+280,[\theta]_{278}-540,[\theta]_{265}-370,[\theta]_{256}-400$, 
$[\theta]_{232}+770$. EIMS (70 eV) $(\mathrm{m} / \mathrm{z})$ (rel. int.): $464[\mathrm{M}]^{+}(86)$, 446(100), 431 (14), $418(25), 403(30), 377(20), 363(35), 350$ (24), 335 (30), 281 (30). HRMS for $\mathrm{C}_{30} \mathrm{H}_{40} \mathrm{O}_{4}$ requires 464.2927. Found 464.2887.

$6 \beta$-Hydroxychamaecydin (3). Orange crystals. Mp $206-208^{\circ} .[\alpha]_{\mathrm{D}}^{25}+100^{\circ}\left(\mathrm{CHCl}_{3} ; c 0.8\right)$. TLC $(6 \%$ EtOAc in hexane) $R_{f}$ 0.42. IR $v_{\max }^{\mathrm{KBr}} \mathrm{cm}^{-1}: 3510,3305,1694,1610$. UV $\lambda_{\max }^{\mathrm{CHCl}_{3}} \mathrm{~nm}(\varepsilon): 348$ (31 000), 339 (25 500), 333 (27 200), 270 (2100), 241 (11600). CD $\left(\mathrm{CHCl}_{3}\right):[\theta]_{453}+1040$. $[\theta]_{426}+40,[\theta]_{416}+100,[\theta]_{396}-260,[\theta]_{378}+105$, $[\theta]_{360}-80,[\theta]_{342}+40,[\theta]_{332}+30,[\theta]_{301}-240,[\theta]_{274}$ $+700,[\theta]_{249}-150$. EIMS $(70 \mathrm{eV}) m / z$ (rel. int.): 464 $\left[\mathrm{M}^{+}(65), 446(100), 431(8), 418(27), 403\right.$ (25), 375 (22), $363(35), 350(22), 335(32), 281$ (15). HRMS for $\mathrm{C}_{30} \mathrm{H}_{40} \mathrm{O}_{4}$ requires 464.2927. Found 464.2945.

$10^{\prime} \beta$-Hydroxycryptoquinone (4). Orange crystals. Mp 210-212 $\quad[\alpha]_{\mathrm{D}}^{25}+40^{\circ} \quad\left(\mathrm{CHCl}_{3} ; \quad c \quad 0.5\right) . \quad$ TLC $\left(\mathrm{CHCl}_{3}\right.$-hexane, $\left.1: 1\right) R_{f}$ 0.30. IR $v_{\max }^{\mathrm{KBr}} \mathrm{cm}^{-1}: 3480,3298$, 1650, 1637. UV $\lambda_{\max }^{\mathrm{CHCl}_{3}} \mathrm{~nm}(\varepsilon): 354$ (29 800), 344 (22800), $340(26500), 270(3200), 240(500)$. EIMS $(70 \mathrm{eV}) \mathrm{m} / z(\mathrm{rel}$. int.): $464\left[\mathrm{M}^{+}(30), 446(100), 402(18), 364(8), 306(40)\right.$, 288 (36). HRMS for $\mathrm{C}_{30} \mathrm{H}_{40} \mathrm{O}_{4}$ requires 464.2927. Found 464.2916.

$10^{\prime} \alpha$-Hydroxycryptoquinone (5). Orange crystals. Mp 202-203. TLC $\left(\mathrm{CHCl}_{3}\right.$-hexane, $\left.1: 1\right) R_{f}$ 0.25. IR $v_{\max }^{\mathrm{KBr}} \mathrm{cm}^{-1}: 3500,3295,1650,1637$. UV $\lambda_{\max }^{\mathrm{CHCl}_{3}} \mathrm{~nm}(\varepsilon): 352$ (29 600), $342(22300), 338$ (26 500), 274 (2200), 240 (510). EIMS (70 eV) $m / z$ (rel. int.): 464 [M] $^{+}$(35), $446(100), 402$ (20), 364 (8), 306 (45), 288 (38). HRMS for $\mathrm{C}_{30} \mathrm{H}_{40} \mathrm{O}_{4}$ requires 464.2927. Found 464.2916 .

$10^{\prime}$-Oxocryptoquinone (6). A soln of the alcohol $4(6 \mathrm{mg})$ in $\mathrm{CH}_{2} \mathrm{Cl}_{2}(3 \mathrm{ml})$ was treated with $\mathrm{MnO}_{2}(5 \mathrm{mg})$. The mixt. was stirred at room temp. $\left(27^{\circ}\right)$ for $3 \mathrm{hr}$, filtered, and the filtrate was concd in vacuo to give the ketone $6(6 \mathrm{mg})$. A sample of 5 ( $3 \mathrm{mg})$ was oxidized by a similar procedure

\section{Note added in proof:}

At the stage of proof, we are aware of a recent report on hexacarbocyclic triterpene quinone methides. Shibuya, T. (1992) Phytochemistry 31, 4289. to give $6(3 \mathrm{mg})$. Orange crystals. $\mathrm{Mp} 182-183^{\circ} .[\alpha]_{\mathrm{D}}^{25}$ $+25^{\circ}\left(\mathrm{CHCl}_{3} ; c\right.$ 0.6). IR $v_{\max }^{\mathrm{KBr}} \mathrm{cm}^{-1}: 3300,1670,1660$, 1640. UV $\hat{\lambda}_{\max }^{\mathrm{CHCl}_{3}} \mathrm{~nm}(\varepsilon): 356$ (29 400), 345 (22600), 340 (26 800), $274(2100), 240(900)$. EIMS $(70 \mathrm{eV}) \mathrm{m} / \mathrm{z}$ (rel. int.): $462[\mathrm{M}]^{+}(100), 447(18), 419(72), 401(13), 391$ (15), 380 (25), 366 (58), 354 (72), 295 (30). HRMS for $\mathrm{C}_{30} \mathrm{H}_{38} \mathrm{O}_{4}$ requires 462.2771 . Found 462.2750 .

Acknowledgment--We are grateful to the National Science Council for financial support (Grant NSC810208-M002-30).

\section{REFERENCES}

1. Gan, W. S. (1958) Manual of Medicinal Plants in Taiwan, Vol. 1, pp 54-55. Nat. Res. Inst. Chin. Med., Taipei.

2. Miura, H., Kawano, N. and Waiss, A. C., Jr (1966) Chem. Pharm. Bull. 14, 1404.

3. Appleton, R. A., McCrindle, R. and Overton, K. H. (1968) Phytochemistry 7, 135.

4. Appleton, R. A., McCrindle, R. and Overton, K. H. (1970) Phytochemistry 9, 581.

5. MacMillan, J. and Walker, E. R. H. (1972) J. Chem. Soc., Perkin Trans. I 981.

6. Shieh, B., lizuka, Y. and Matsubara, Y. (1981) Agric. Biol. Chem. 45, 1493.

7. Yatagai, M. and Sato, T. (1986) Biochem. Syst. Ecol 14, 469.

8. Matsui, T., Ohishi, H. and Nakayama, N. (1989) Kogakubu Kenkyu Hokoku 35, 157.

9. Vernin, G., Faure, R. and Pieribattesti, J. C. (1990) J. Essent. Oil Res. 2, 211.

10. Hirose, Y., Hasegawa, S. and Ozaki, N. (1983) Tetrahedron Letters 24, 1535. 